\title{
Direct Observation of Stochastic Domain-Wall Depinning in Magnetic Nanowires
}

\author{
Mi-Young Im, ${ }^{1 *}$ Lars Bocklage, ${ }^{2}$ Peter Fischer, ${ }^{1}$ and Guido Meier ${ }^{2}$ \\ ${ }^{1}$ Center for X-ray Optics, Lawrence Berkeley National Laboratory, Berkeley CA94720, \\ USA \\ ${ }^{2}$ Institut für Angewandte Physik und Zentrum für Mikrostrukturforschung, Universität \\ Hamburg, Jungiusstrasse 11, 20355 Hamburg, Germany
}

\begin{abstract}
:
The stochastic field-driven depinning of a domain wall pinned at a notch in a magnetic nanowire is directly observed using magnetic X-ray microscopy with high lateral resolution down to $15 \mathrm{~nm}$. The depinning-field distribution in $\mathrm{Ni}_{80} \mathrm{Fe}_{20}$ nanowires considerably depends on the wire width and the notch depth. The difference in the multiplicity of domain-wall types generated in the vicinity of a notch is responsible for the observed dependence of the stochastic nature of the domain wall depinning field on the wire width and the notch depth. Thus the random nature of the domain wall depinning process is controllable by an appropriate design of the nanowire.
\end{abstract}


For concepts of logic and storage devices utilizing magnetic domain-wall (DW) displacement along a nanowire [1-5] one of the fundamental issues is the precise control of DW motion. The latter is directly linked to the reproducibility of DW propagation, pinning, and depinning. Tunable and repeatable DW motion is important for achieving high performance in DW logic and memory devices [6-9], and the stochastic nature of DW motion is a major challenge to be overcome to apply the scheme of DW motion to next generation memory technologies [10-11]. One attempt to control the DW motion is to manufacture artificial trapping sites within magnetic nanowires [12-17]. Experimental studies on DW dynamics about artificial trapping sites reported so far have been performed by indirect probes like macroscopic hysteresis loops and magnetoresistance measurements $[13,14,18,19]$. Moreover, the few direct observations have not focussed on the in-depth investigation of the stochastic behavior of DW motion around trapping sites in magnetic nanowires [17, 20]. Thus, statistical observation of DW propagation in the vicinity of artificial trapping sites in nanowires together with the experimental clarification of the stochastic nature of DW motion have yet remained a scientific challenge.

In this Letter, we report the direct observation of the stochastic behavior of the DW depinning field in notch-patterned $\mathrm{Ni}_{80} \mathrm{Fe}_{20}$ (permalloy) nanowires with different wire widths $(w)$, notch depths $\left(\mathrm{N}_{\mathrm{d}}\right)$, and film thicknesses $(\mathrm{t})$ using magnetic transmission soft X-ray microscopy (MTXM) with a lateral resolution of $15 \mathrm{~nm}$ obtained by recent achievements in Fresnel zone plate technology [21]. The MTXM beamline (6.1.2) used to observe the evolution of magnetic DWs is installed at the Advanced Light Source in Berkeley, CA. The experimental setup of this X-ray microscope is described elsewhere in detail [22]. A condensor zone plate (CZP) with a pinhole close to the sample acts as a linear monochromator, which provides selective X-rays, e.g., with an energy corresponding to the $\mathrm{Ni}_{3}(854 \mathrm{eV})$ or $\mathrm{Fe}_{3}(706 \mathrm{eV})$ absorption edge. Magnetic contrast in MTXM is provided by the X-ray magnetic circular dichroism [23]. Magnetic imaging of the permalloy nanowires was performed in an in-plane geometry where the wires are mounted under an angle of $60^{\circ}$ with respect to the photon beam direction and 
parallel to the magnetic field. To study DW motion magnetic images in nanowires are recorded with varying external magnetic field generated by a solenoid with field strengths of up to $\pm 100 \mathrm{mT}$. The fine magnetic contrast without structural contrast is accomplished by normalization of an image taken at a particular field using an image obtained at a saturation field. The nanowires are prepared on $100 \mathrm{~nm}$ thick siliconnitride membranes by electron-beam lithography and thermal evaporation. For protection of the permalloy $2 \mathrm{~nm}$ aluminum is sputtered, which is oxidized in a pure oxygen atmosphere.

Typical structural images of the wires with triangular notches measured by scanningelectron microscopy (SEM) are shown in Figs. 1(a) to 1(c). Magnetic images are taken at the $\mathrm{Fe} \mathrm{L}_{3}$ absorption edge. The wires are initially saturated to positive $\mathrm{x}$ direction and then a reversed field is applied to trigger the nucleation and propagation of a magnetic DW along the wire. DW evolution images for $50 \mathrm{~nm}$ thick wires with widths of $\mathrm{w}=150$, 250, and $450 \mathrm{~nm}$ are demonstrated in Figs. 1(d) to 1(f), where the notch depth is about $50 \%$ of the width of each wire. As demonstrated by the experiments shown in Figs. 1(d) to 1(f) the magnetic DW is created within the elliptical pad due to its lower shape anisotropy compared to the narrow wire. By increasing the field strength the DW propagates towards the notch. The DW evolution process observed between the pad and the notch is overall identical in all wires with different widths and pad sizes, even though the wire of width $\mathrm{w}=450 \mathrm{~nm}$ with a relatively wide neck shows the distinction that the DW starts leaving toward the notch before the magnetization reversal of the elliptical pad is fully completed. The DW is pinned at a notch due to the pinning force exerted by this notch and the pinned DW is a tail-to-tail wall considering saturation $(+\mathrm{x})$ and reversing $(-\mathrm{x})$ field direction [15]. When the external force acting on the pinned DW overcomes the pinning force by the notch, the DW is depinned and propagates to the sharp tip at the right end of the wire where it is annihilated. One can see in Figs. 1(d) to $1(\mathrm{f})$ that the DW depinning field decreases with increasing wire width, which is the consequence of different sizes of DWs governed by different wire widths. The size of the DW trapped at a notch grows as the wire is widened. A bigger DW is energetically 
unfavorable rather than a smaller DW, thus the DW depinning field reduces with increasing wire width $[13,14,16,19]$.

To investigate the statistical behavior of the DW depinning field at a notch, we recorded magnetic images in successive hysteretic reversal cycles starting at a fully saturated state of the wire. In repeated measurements we found that the pinning probability of a DW is a function of the wire width. The probability is decreased from about 92 to $75 \%$ of the total number of repeated measurements as the wire narrows from 450 to $250 \mathrm{~nm}$. Three representative DW evolution image sequences for wires of $\mathrm{w}=150,250$, and $450 \mathrm{~nm}$ are shown in Fig. 2(a). Here, the colors from red to blue indicate the DW pinning and depinning-field strenghts. Within repeated experiments carried out at the same wire under identical measurement conditions the DW depinning field shows stochastic behavior, as visualized by various colors. Considering that the depinning field of a DW in a magnetic nanowire is strongly correlated with the DW structure [12-14, 24], the stochastic nature of the DW depinning field can be interpreted to be induced by the generation of various DW types in the vicinity of a notch. We observed that DWs with different micromagnetic structures are depinned at different fields. Figure 2(b) presents detailed pictures of DWs at a notch in the wire of $\mathrm{w}=250$ $\mathrm{nm}$ and $\mathrm{N}_{\mathrm{d}} \sim 50 \%$, which reveals that depinning fields are related to the various DW structure. Another notable feature observed in repeated experiments is that the depinning field appears different even though the DW pinning field is identical, which implies that the DW depinning process is not subordinate to the DW pinning mechanism. This result suggests that complicated phenomena around a notch like the creation of different DW structures and the interaction between the DW and the notch plays the dominant role in the DW depinning process $[12,13]$.

The stochastic nature of the DW depinning field for different notch depths and wire widths has been systematically investigated by determination of the DW depinning-field distribution from depinning events taken in repeated experiments at least 40 times for each wire. The depinning field is measured by sweeping the external field in steps of about $0.5 \mathrm{mT}$. In Fig. 3(a) the depinning-field distribution for $50 \mathrm{~nm}$ thick wires of 
$\mathrm{N}_{\mathrm{d}} \sim 50 \%$ with widths of $\mathrm{w}=150,250$, and $450 \mathrm{~nm}$ and for wires of $\mathrm{N}_{\mathrm{d}} \sim 30 \%$ with $\mathrm{w}=250 \mathrm{~nm}$ are plotted. It can be seen in Fig. 3(a) that the width of the DW depinningfield distribution is found to depend on the wire width and the notch depth. The DW depinning field is widely distributed in wires of width $\mathrm{w}=250 \mathrm{~nm}$ compared to wires of widths $\mathrm{w}=150$ and $450 \mathrm{~nm}$. The depinning-field distribution for wire with widths of $\mathrm{w}=250 \mathrm{~nm}$ becomes narrow as the notch depth decreases from 50 to $30 \%$. We also investigated the statistical distribution of DW depinning fields for wires with a thickness of $30 \mathrm{~nm}$. Depinning-field distributions for wires of $\mathrm{N}_{\mathrm{d}} \sim 50 \%$ with $\mathrm{w}=250 \mathrm{~nm}$ and wires of $\mathrm{N}_{\mathrm{d}} \sim 30 \%$ with $\mathrm{w}=150,250$, and $450 \mathrm{~nm}$ are displayed in Fig. 3(b). In the $30 \mathrm{~nm}$ thick wires, we focussed on the wires with $\mathrm{N}_{\mathrm{d}} \sim 30 \%$ instead of $\mathrm{N}_{\mathrm{d}} \sim 50 \%$ based on the experimental result for the $50 \mathrm{~nm}$ thick wire where the wire with $\mathrm{N}_{\mathrm{d}} \sim 30 \%$ exhibits a narrow distribution, which is more preferred for application in DW devices. A similar trend of the influence of the width on the DW depinning-field distribution is also witnessed in the $30 \mathrm{~nm}$ thick wire as shown in Fig. 3(b). It is worth pointing out that in Fig. 3 isolated dominant peaks exist in the distributions. This result suggests that the thermal effect on the DW depinning process is not the major cause of the observed fluctuation of DW depinning field, since a Gaussian statistical distribution is expected for thermally activated DW depinning $[15,25]$.

To quantitatively examine the degree of stochastic nature of the DW depinning field with varying wire width and notch depth, we have determined the standard deviation $\sigma$ of the DW depinning field from statistical analysis of repeated measurements. The standard deviation of the DW depinning field indicates the degree of depinning-field fluctuation. Figure 4 shows the standard deviations as a function of the wire width (a) and the notch depth (b) for wires of $t=30$ and $50 \mathrm{~nm}$. In the case of a $150 \mathrm{~nm}$ wide wire with $\mathrm{N}_{\mathrm{d}} \sim 50 \%$ and a thickness of $50 \mathrm{~nm}$, the standard deviation of the depinning field is minimized to below $0.7 \mathrm{mT}$. In the case of a $250 \mathrm{~nm}$ wide wire with $\mathrm{N}_{\mathrm{d}} \sim 50 \%$ and a thickness of $30 \mathrm{~nm}$, the standard deviation of the depinning field is as high as $\sim 5.5 \mathrm{mT}$. Figure 4 demonstrates that the standard deviation depends sensitively on the wire width and the notch depth, which implies that the stochastic nature of the DW depinning field 
is decisively influenced by the geometry of the wire. The alteration of the stochastic nature of the DW depinning field with respect to wire width and notch depth is found to be a general tendency irrespective of the wire thickness in permalloy wires, although there are slight differences in the absolute value and the variation rate of the standard deviation with the wire width and the notch depth. Considering that the depinning field of the DW is strongly related to the DW structure and thus the stochastic nature of the DW depinning field is governed by the number of different DW structures that can be generated in a wire, the stochastic nature of the DW depinning field witnessed in the present experiments is presumably caused by the diversity of generable DW structures in the vicinity of a notch. In wires exhibiting narrower fluctuations of the DW depinning field less types of DWs are accessible compared to wires with strongly fluctuating depinning fields. Thus, we conclude that the key to obtain a reproducible DW depinning process is a single DW type at a notch, which can be achieved by a proper selection of the wire and notch geometry.

Our experiments report the direct observation of the stochastic nature of the DW depinning field at a notch in permalloy wires. We find that the stochastic nature of the DW depinning field depends on the wire width and the notch depth. The number of DW types generated in the vicinity of a notch wire has a strong impact on the random nature of the DW depinning field. Our results clearly demonstrate that the stochastic nature of the DW depinning process can be minimized by proper geometrical design of the wires.

\section{Acknowledgements}

This work was supported by the Director, Office of Science, Office of Basic Energy Sciences, of the U.S. Department of Energy under Contract No. DE-AC02-05CH11231. Financial support of the Deutsche Forschungsgemeinschaft via SFB 668 "Magnetism from the Single Atom to the Nanostructure" and via Graduiertenkolleg 1286 "Functional Metal-Semiconductor Hybrid Systems" is gratefully acknowledged. 


\section{References}

[1] D. A. Allwood, G. Xing, M. D. Cooke, C. C. Faulkner, D. Atkinson, N. Vernier, and R. P. Cowburn, Science 296, 2003 (2002).

[2] S. S. P. Parkin, U. S. Patent 6834005 (2004).

[3] R. P. Cowburn, Nature 448, 544 (2007).

[4] T. Ono, H. Miyajima, K. Shigeto, K. Mibu, N. Hosoito, and T. Shinjo, Science 84, 48 (1999).

[5] D. Atkinson, D. A. Allwood, G. Xiong, M. D. Cooke, C. C. Faulkner, and R. P. Cowburn, Nature 2, 85 (2003).

[6] M. Kläui, C. A. F. Vaz, J. Rothman, J. A. C. Bland, W.Wernsdorfer, G. Faini, and E. Cambril, Phys. Rev. Lett. 90, 097202 (2003).

[7] M. Kläui, C. A. F. Vaz, J. A. C. Bland, W.Wernsdorfer, G. Faini, E. Cambril, L. J. Heyderman, F. Nolting, and U. Rüdiger, Phys. Rev. Lett. 94, 106601 (2005).

[8] M. Hayashi, L. Thomas, R. Moriya, C. Rettner, and S. S. P. Parkin, Science 320, 209 (2008).

[9] A. J. Zambano and W. P. Pratt, Jr., Appl. Phys. Lett. 85, 1562 (2004).

[10] G. Meier, M. Bolte, R. Eiselt, B. Krüger, D.-H. Kim, and P. Fischer, Phys. Rev. Lett. 98, 187202 (2007).

[11] M.-Y. Im, S.-H. Lee, D.-H. Kim, P. Fischer, and S.-C. Shin, Phys. Rev. Lett. 100, 167204 (2008).

[12] M. Hayashi, L. Thomas, C. Rettner, R. Moriya, X. Jiang, and S. S. P. Parkin, Phys. Rev. Lett. 97, 207205 (2006).

[13] C. C. Faulkner, M. D. Cooke, D. A. Allwood, D. Petit, D. Atkinson, and R. P. Cowburn, J. Appl. Phys. 95, 6717 (2004).

[14] M. Kläui, H. Ehrke, U. Rüdiger, T. Kasama, R. E. Dunin-Borkowski, D. Backes, L. J. Heyderman, C. A. F. Vaz, J. A. C. Bland, G. Faini, E. Cambril, and W.Wernsdorfer, Appl. Phys. Lett. 87, 102509 (2005).

[15] P. Lendecke, R. Eiselt, G. Meier, and U. Merkt, J. Appl. Phys. 103, 073909 (2008).

[16] A. Himeno, T. Okuno, T. Ono, K. Mibu, S. Nasu, and T. Shinjo, J. Magn. Magn. Mater. 286, 167 (2005).

[17] M. Herrmann, S. McVitie, and J. N. Chapman, J. Appl. Phys. 87, 2994 (2000).

[18] W. Kleemann, J. Rhensius, O. Petracic, J Ferré, and J. P. Jamet, Phys. Rev. Lett. 99, 097203 (2007).

[19] Y. Yokoyama, Y. Suzuki, S. Yuasa, K. Ando, K. Shigeto, T. Shinjo, P. Gogol, J. Miltat, A. Thiaville, T. Ono, and T. Kawagoe, J. Appl. Phys. 87, 5618 (2000). 
[20] L. Thomas, C. Rettner, M. Hayashi, M. G. Samant, and S. S. P. Parkin, Appl. Phys. Lett. 87, 262501 (2005).

[21] W. Chao, B. H. Harteneck, J. A. Liddle, E. H. Anderson, and D. T. Attwood, Nature 435, 1210 (2005).

[22] P. Fischer, D.-H. Kim, W. Chao, J. A. Liddle, E. H. Anderson, and D. T. Attwood, Materials Today 9, 26 (2006).

[23] G. Schütz, W. Wagner, W. Wilhelm, P. Kienle, R. Zeller, R. Frahm, and G. Materlik, Phys. Rev. Lett. 58, 737 (1987).

[24] R. Skomski and J. M. D. Coey, Permanent Magnetism (Institute of Physics, Bristol, 1999).

[25] E. Martinez, L. Lopez-Diaz, O. Alejos, L. Torres, and C. Tristan, Phys. Rev. Lett. 98, 267202 (2007). 


\section{Figure Captions}

Fig. 1. (a) Typical SEM image of a $50 \mathrm{~nm}$ thick nanowire with a width of $150 \mathrm{~nm}$ together with enlarged notch patterns with notch depths of about $30 \%$ (b) and about $50 \%$ (c) of the wire width. Three representative image sequences of magnetic domain wall evolution along the hysteresis cycle for wire widths of $w=150 \mathrm{~nm}(\mathrm{~d}), 250 \mathrm{~nm}$ (e), and $450 \mathrm{~nm}$ (f). The magnetic field of the DW evolution pattern is indicated on the lower right.

Fig. 2. (a) Domain wall evolution patterns taken from three consecutive experiments under identical measurement conditions for wires of width $\mathrm{w}=150,250$, and $450 \mathrm{~nm}$. The color scale represents the field when a domain wall is pinned and depinned at a notch. (b) Domain-wall structures for the wire of $\mathrm{w}=250 \mathrm{~nm}$ and $\mathrm{N}_{\mathrm{d}} \sim 50 \%$ observed in the vicinity of a notch right before the depinning.

Fig. 3. Distributions of domain wall depinning fields for (a) $50 \mathrm{~nm}$ thick and (b) $30 \mathrm{~nm}$ thick nanowires determined from depinning fields taken by repeated experiments with at least 40 repetitions for each wire under identical measurement conditions.

Fig. 4. Standard deviations of the domain wall depinning field with respect to (a) wire width and (b) notch depth. The standard deviation of the depinning field was taken from the statistical analysis of more than 30 DW depinning fields for each wire. 


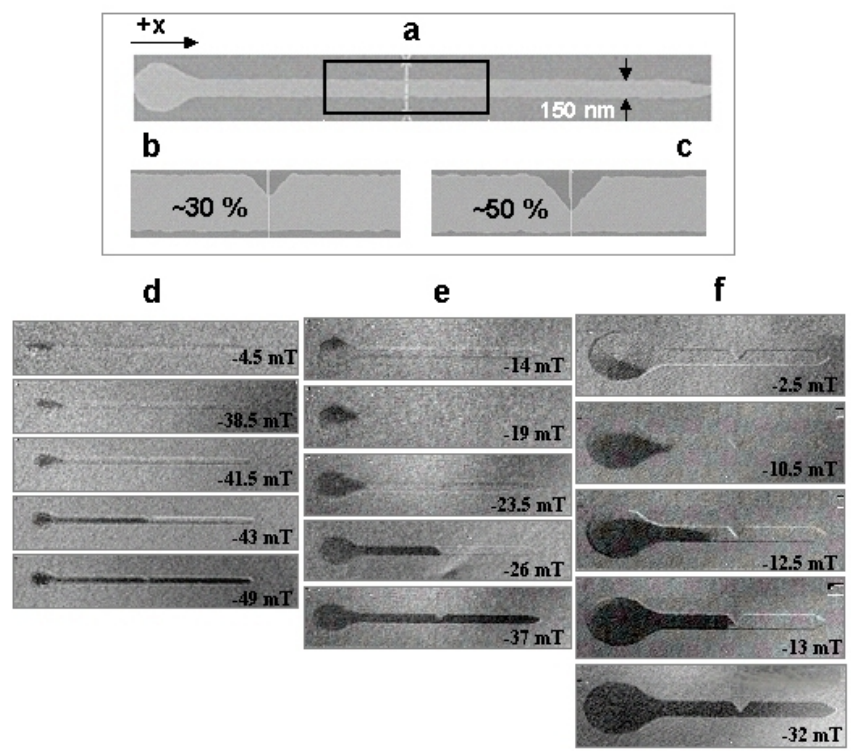

Fig. 1 


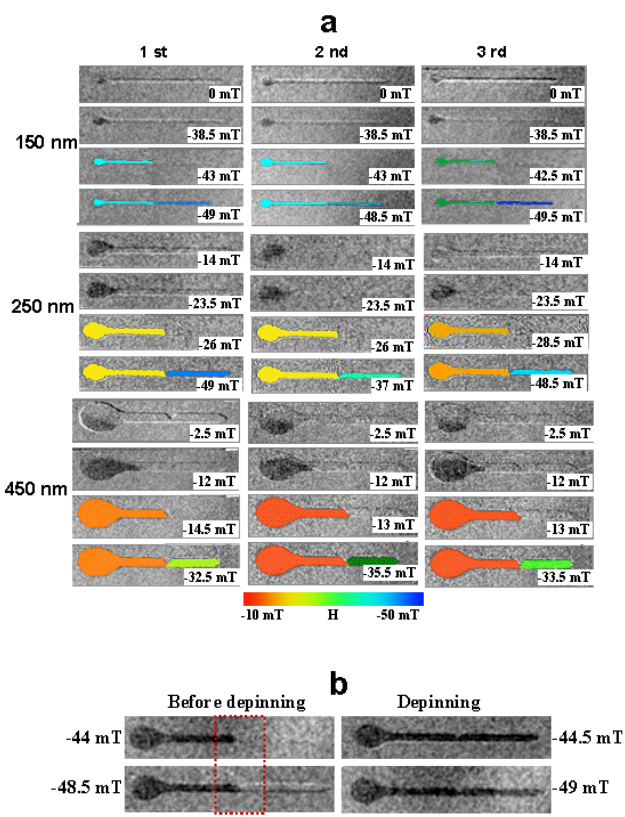

Fig. 2 

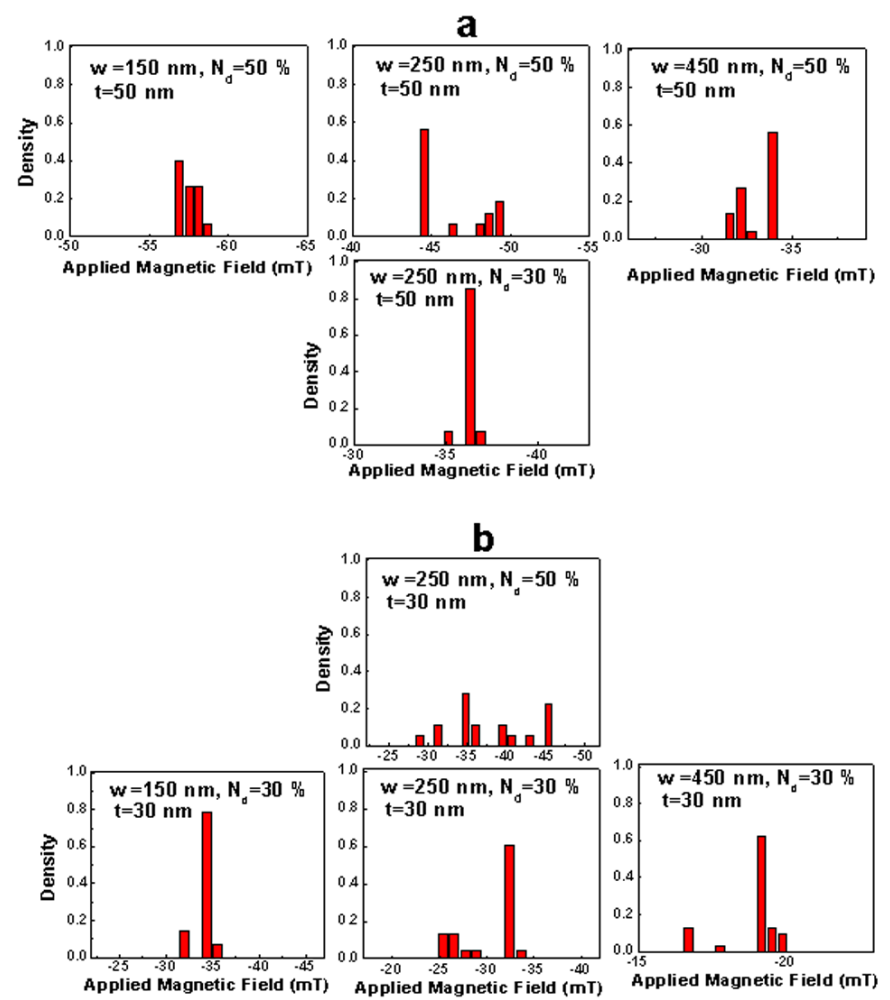

Fig. 3 


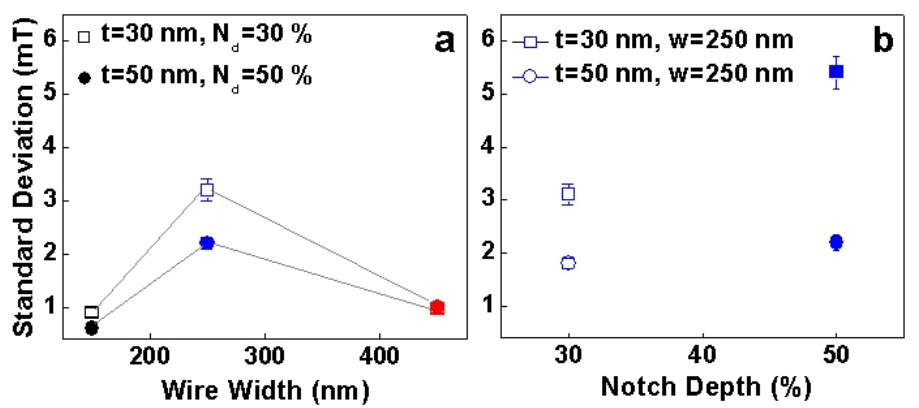

Fig. 4 